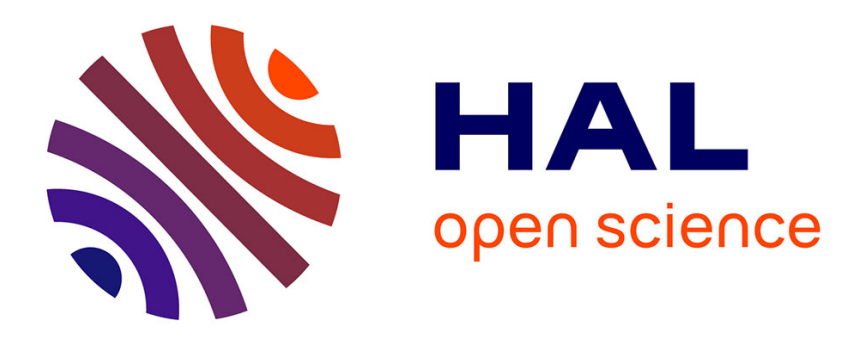

\title{
On the controllability of the hydrostatic Stokes equations
}

Sergio Guerrero, Francisco Guillen - Gonzalez

\section{To cite this version:}

Sergio Guerrero, Francisco Guillen - Gonzalez. On the controllability of the hydrostatic Stokes equations. 2006. hal-00120195

\section{HAL Id: hal-00120195 \\ https://hal.science/hal-00120195}

Preprint submitted on 13 Dec 2006

HAL is a multi-disciplinary open access archive for the deposit and dissemination of scientific research documents, whether they are published or not. The documents may come from teaching and research institutions in France or abroad, or from public or private research centers.
L'archive ouverte pluridisciplinaire HAL, est destinée au dépôt et à la diffusion de documents scientifiques de niveau recherche, publiés ou non, émanant des établissements d'enseignement et de recherche français ou étrangers, des laboratoires publics ou privés. 


\title{
On the controllability of the hydrostatic Stokes equations
}

\author{
S. Guerrero* and F. Guillén-González ${ }^{\dagger}$
}

\begin{abstract}
This paper is devoted to present some results on the controllability of the hydrostatic Stokes equations. The first main result of this paper states that the approximate null controllability of this system holds. This is proved whatever the boundary conditions are. Then, we extend this result to an exact null controllability result when the boundary conditions are $\partial_{z} u=0$ (the vertical derivative of the horizontal velocity) at the bottom.
\end{abstract}

\section{Introduction and main results}

The main purpose of this article is to study some controllability problems, either approximate controllability or exact controllability, for the three-dimensional hydrostatic Stokes equations, which is the linear problem associated to the hydrostatic Navier-Stokes equations (also called Primitive Equations).

The Primitive Equations stand for one of the most fundamental governing equations in the field of geophysical fluids, for atmospheric and oceanic flows. This system is formally derived from the $3 D$ incompressible Navier-Stokes equations for the case of large time and horizontal spatial scales $([1,13])$. In fact, the Primitive Equations of large scale oceanic flow results as the asymptotic limit of the Navier-Stokes model as the aspect ratio of the vertical to the horizontal length scale goes to zero $([4,5])$. In particular, this model can describe the general circulation of the water in lakes and oceans [12].

Control problems of fluids have extensively been studied in the last years (see, for instance, [3], [7] and [9], where the model used is the Navier-Stokes system). As an extension of this, one can also consider control problems associated to geophysical fluids. As far as we know, the controllability results we will present below are the first ones concerning geophysical fluids.

For simplicity, we take constant density, cartesian coordinates $\left(x=\left(x_{1}, x_{2}\right)\right.$ in the horizontal direction and $z$ perpendicular to the surface $z=0$ ) and we

\footnotetext{
*Université Pierre et Marie Curie-Paris6, UMR 7598 Laboratoire Jacques-Louis Lions, Paris, F-75005 France. E-mail: guerrero@ann.jussieu.fr.

†Departamento de Ecuaciones Diferenciales y Análisis Numérico, Universidad de Sevilla, Aptdo. 1160, 41080 Sevilla, Spain. E-mail: guillen@us.es.
} 
assume that the effects due to temperature and salinity can be decoupled from the flow dynamics.

The most distinguished feature of the Primitive Equations is that the hydrostatic balance $\partial_{z} p=-\rho g$ (being $p$ the $3 D$ pressure, $\rho$ the density and $g$ the gravity acceleration) replaces the momentum equation for the vertical velocity $v$. Since we have assumed constant density, this balance implies $p(x, z, t)=$ $p_{S}(x, t)-\rho g z$, where $p_{S}$ is a $2 D$ function defined in the horizontal plane (the surface pressure).

Let $T>0$, let $S$ be an open connected regular set of $\mathbf{R}^{2}$ (the surface) and let $D: S \rightarrow \mathbf{R}_{+}$be a regular function (the bottom function). We will specify the regularity of $S$ and $D$ below. Then, we introduce the oceanic domain

$$
\Omega=\left\{(x, z)=\left(x_{1}, x_{2}, z\right) \in \mathbf{R}^{3}: x \in S, z \in(-D(x), 0)\right\} .
$$

We design by $\Gamma_{S}$ the surface boundary

$$
\Gamma_{S}=\left\{(x, z) \in \mathbf{R}^{\mathbf{3}}: x \in S, z=0\right\}=S \times\{0\},
$$

by $\Gamma_{l}$ the lateral boundary

$$
\Gamma_{l}=\left\{(x, z) \in \mathbf{R}^{3}: x \in \partial S, z \in(-D(x), 0)\right\}
$$

and by $\Gamma_{b}$ the bottom boundary

$$
\Gamma_{b}=\left\{(x, z) \in \mathbf{R}^{3}: x \in S, z=-D(x)\right\} .
$$

We will denote $\Sigma_{S}:=\Gamma_{S} \times(0, T), \Sigma_{l}:=\Gamma_{l} \times(0, T)$ and $\Sigma_{b}:=\Gamma_{b} \times(0, T)$.

We also consider an open subset $\omega \subset \Omega$ (the control domain) which can be assumed to be as small as desired. Let us set

$$
Q:=\Omega \times(0, T) .
$$

Let us denote by $u=\left(u_{1}, u_{2}\right): Q \rightarrow \mathbf{R}^{2}$ the horizontal velocity, $v: Q \rightarrow \mathbf{R}$ the vertical velocity and $p_{S}$ the surface pressure. We will work with the following system:

$$
\begin{cases}u_{t}-\Delta u-\partial_{z z}^{2} u+\alpha\left(x_{2}\right) u^{\perp}+\nabla p_{S}=h 1_{\omega} & \text { in } Q, \\ \nabla \cdot u+\partial_{z} v=0 & \text { in } Q, \\ u=0 & \text { on } \Sigma_{l}, \\ \partial_{z} u=0, \quad v=0 & \text { on } \Sigma_{S}, \\ B(u)=0, \quad(u, v) \cdot n_{b}=0 & \text { on } \Sigma_{b}, \\ u(\cdot, 0)=u^{0} & \text { in } \Omega .\end{cases}
$$

Here, the term $\alpha\left(x_{2}\right) u^{\perp}=\alpha\left(x_{2}\right)\left(-u_{2}, u_{1}\right)$ is the Coriolis force which represents the influence of the rotation of the earth (for more details on the obtention 
of this term from the Navier-Stokes equations when the aspect ratio tends to zero, see [4]) and $h$ stands for the control function. Observe also that the differential operators are taken with respect to the $x$-variables (the differentiation with respect to $z$ will always be specified). Moreover, the operator $B$ pretends to express various boundary conditions which will be consider in this paper. Precisely, we are interested in homogeneous

$$
\left\{\begin{array}{l}
\operatorname{Dirichlet}(w=0), \\
\operatorname{Neumann}\left(\partial_{n} w=0\right), \\
\operatorname{vertical}\left(\partial_{z} w=0\right)
\end{array}\right.
$$

boundary conditions. Finally, $n_{b}$ stands for the outward unit normal to $\Gamma_{b}$.

We remark that the boundary conditions considered in (1) are the natural ones (see, for instance, [13] and [12]).

Observe that the vertical integral of the horizontal velocity is divergencefree (i.e. $\nabla \cdot \int_{-D(x)}^{0} u d z=0$ ), due to the incompressibility of the flow and the boundary conditions on the vertical velocity at the surface and bottom. Then, it is shown in [12] that the surface pressure is the Lagrange multiplier of this new constraint and the dissipativity of this system holds; both things drive to the existence of a weak solution. The study of the regularity of system (1) is much more complicated, due to the singularity of the domain under consideration. Existence and uniqueness of a strong solution of the linear stationary problem associated to (1) is proved in [16] as $\log$ as $D(x)>0$ in $\bar{S}$. This result can be readily extended to the evolution problem $(1)$.

In this paper the following regularity for the elements which define the oceanic domain are imposed:

$$
S \in W^{3, \infty}, D \in W^{2, \infty}(S) \text { and } D(x)>0 \text { in } \bar{S} .
$$

We remark here that the regularity condition on $S$ could be weakened to be $W^{1, \infty}$, but we have chosen to do direct proofs which in particular require $H^{4}$-regularity for a Stokes-like problem posed in $S$ (see (53) below). On the other hand, the last assumption in (3) means that our domain has sidewalls.

Furthermore, we will use that

$$
\alpha \in L^{\infty}(\mathbf{R}) .
$$

In fact, $\alpha\left(x_{2}\right)=2 f \sin \left(\ell\left(x_{2}\right)\right)$, where $f$ is the module of the Coriolis force and $\ell$ is the latitude.

The first main result of this paper concerns the approximate null controllability of system (1). It is given in the following theorem:

Theorem 1 Let (3) and (4) hold and let $B$ denote one of the three conditions given in (2). Then, for each $\varepsilon>0$, there exists a control $v \in L^{2}(\omega \times(0, T))$ such that the solution $(u, v)$ of (1) satisfies

$$
\|(u, v)(\cdot, T)\|_{L^{2}(\Omega)} \leq \varepsilon .
$$


In order to prove this theorem, we use the classical approach as long as a linear control system is concerned. Namely, we consider the associated adjoint system:

$$
\begin{cases}-\psi_{t}-\Delta \psi-\partial_{z z}^{2} \psi-\alpha\left(x_{2}\right) \psi^{\perp}+\nabla \beta_{S}=0 & \text { in } Q, \\ \nabla \cdot \psi+\partial_{z} \gamma=0 & \text { in } Q, \\ \psi=0 & \text { on } \Sigma_{l}, \\ \partial_{z} \psi=0, \quad \gamma=0 & \text { on } \Sigma_{S}, \\ B^{*}(\psi)=0, \quad(\psi, \gamma) \cdot n_{b}=0 & \text { on } \Sigma_{b}, \\ \psi(\cdot, T)=\psi^{0} & \text { in } \Omega .\end{cases}
$$

Here, $(\psi, \gamma)$ is the velocity vector field, $\beta_{S}$ is the surface pressure, $B^{*}$ is the (formal) adjoint operator of $B$ and $\psi^{0}$ is the initial horizontal velocity. Observe that this system is backwards in time.

Then, property (5) is equivalent to: for any $\psi^{0} \in L^{2}(\Omega)$, we have

$$
\psi=0 \text { in } \omega \times(0, T) \Rightarrow(\psi, \gamma)=0 \text { in } \Omega \times(0, T) .
$$

This fact is classical and can be found, for instance, in [11].

Remark 1 In the proof of this theorem, we will see that a stronger result holds, namely $(7)$ is deduced for any $(\psi, \gamma)$ satisfying the equations

$$
\begin{gathered}
-\psi_{t}-\Delta \psi-\partial_{z z}^{2} \psi-\alpha\left(x_{2}\right) \psi^{\perp}+\nabla \beta_{S}=0 \text { in } Q, \\
\nabla \cdot \psi+\partial_{z} \gamma=0 \text { in } Q,
\end{gathered}
$$

and the boundary conditions

$$
\begin{aligned}
& \psi=0 \text { on } \Sigma_{l}, \\
& \gamma=0 \text { on } \Sigma_{S}
\end{aligned}
$$

and

$$
(\psi, \gamma) \cdot n_{b}=0 \text { on } \Sigma_{b}
$$

That is, the boundary condition $\partial_{z} \psi=0$ on $\Sigma_{S}$ and $B^{*}(\psi)=0$ on $\Sigma_{b}$ are not necessary.

The second main result of this paper concerns the exact null controllability of system (1):

Theorem 2 Let (3) hold and let $B u=\partial_{z} u$. Then, there exists a control $v \in$ $L^{2}(\omega \times(0, T))$ such that the solution $(u, v)$ of $(1)$ satisfies

$$
(u, v)(\cdot, T)=0 \text { in } \Omega
$$


We also follow a classical approach to prove Theorem 2. Let $\left(\psi, \gamma, \beta_{S}\right)$ be the solution of $(6)$ with $B^{*}(\psi)=-\partial_{z} \psi$. Then, it is well-known that the null controllability of (1) with $L^{2}$-controls is equivalent to an observability estimate for $\left(\psi, \gamma, \beta_{S}\right)$. More precisely, we will prove the existence of a constant $C>0$ such that

$$
\|(\psi, \gamma)(\cdot, 0)\|_{H^{1}(\Omega) \times L^{2}(\Omega)}^{2} \leq C \iint_{\omega \times(0, T)}|\psi|^{2} d x d z d t,
$$

for any $\psi^{0} \in L^{2}(\Omega)$. The proof of this inequality follows the ideas first introduced in [7], [8] and then used in [9] and [3].

Remark 2 To our best knowledge, the previous theorems are the first controllability results for the hydrostatic Stokes system. On the other hand, there are two exact controllability problems which we consider particularly interesting:

- The case where another boundary conditions are considered at the bottom. Observe that in Theorem 2 we have imposed that $\partial_{z} u=0$ at the bottom but both Dirichlet and Neumann boundary conditions could also be considered. In the proof of Theorem 2, it will be seen that the fact that the function $\partial_{z} u$ fulfills a complete problem (as seen in (38)) is essential in our argument. This is not the case when Dirichlet and Neumann boundary conditions are imposed at the bottom.

- The local exact controllability of the hydrostatic Navier-Stokes system. This relies on the study of the exact null controllability of a linearized problem, which turns to be much more involved than ours.

This paper is organized as follows: in the second section, we will present some technical results which will be used all along the paper. In Section 3 and Section 4, we prove Theorem 1 and Theorem 2, respectively while the last section is an appendix devoted to prove a Carleman inequality which will be used in the proofs of both theorems.

\section{Technical results}

\subsection{Regularity result for a Stokes type problem}

Let $U \in \mathbf{R}^{\mathbf{N}}$ be a $W^{3, \infty}$ open set and $a, A$ and $m$ be three functions satisfying

$$
a, A \in L^{\infty}(U \times(0, T)) \text { and } m \in W^{2, \infty}(U) .
$$

Let us also denote $Q_{0}:=U \times(0, T)$ and $\Sigma_{0}=\partial U \times(0, T)$ We consider the following Stokes-type problem:

$$
\begin{cases}w_{t}-\Delta w+a(x, t) w^{\perp}+A(x, t) \cdot \nabla w+m(x) \nabla p=f & \text { in } Q_{0}, \\ \nabla \cdot w=0 & \text { in } Q_{0}, \\ w=0 & \text { on } \Sigma_{0} \\ w(\cdot, 0)=w^{0} & \text { in } U .\end{cases}
$$


Here, $w^{\perp}$ stands for $\left(-w_{2}, w_{1}\right)$ when $N=2$ and for $w$ when $N>2$. For our purposes, we will just need to apply this result with $N=2$ and $a$ and $A$ functions independent of the $t$ variable.

Lemma 1 Assume that the coefficients $a, A$ and $m$ satisfy (9). Let $w^{0} \in$ $H_{0}^{1}(U), f \in L^{2}\left(Q_{0}\right)$ and let us suppose that there exists two positive constants $m_{1}, m_{2}$ such that $m_{1} \leq m(x) \leq m_{2}$ in $U$. Then, there exists a unique solution $(w, p)$ of $(10)$ which belongs to the space

$\left(L^{2}\left(0, T ; H^{2}(U)\right) \cap H^{1}\left(0, T ; L^{2}(U)\right)\right) \times L^{2}\left(0, T ; H^{1}(U)\right):=X \times L^{2}\left(0, T ; H^{1}(U)\right)$.

Moreover, there exists a positive constant $C$ such that

$$
\|(w, p)\|_{X \times L^{2}\left(0, T ; H^{1}(U)\right)} \leq C\left(\left\|w^{0}\right\|_{H^{1}(U)}+\|f\|_{L^{2}\left(Q_{0}\right)}\right) .
$$

Proof: Even if the idea we will follow here is classical in this kind of systems, there is the novelty of the presence of a given function $m(x)$ multiplying the pressure term. We will first deduce the existence of a (unique) energy solution $w \in L^{2}\left(0, T ; H^{1}(U)\right) \cap L^{\infty}\left(0, T ; L^{2}(U)\right)$, then we will prove that the time derivative of this function belongs to $L^{2}\left(Q_{0}\right)$ and finally we will regard (10) as a stationary Stokes-type system with right hand side in $L^{2}\left(Q_{0}\right)$.

Let us define the new variable $q=\frac{1}{m(x)} w$, which satisfies

$$
\begin{cases}m(x) q_{t}-\nabla \cdot(m(x) \nabla q)+(a m)(x, t) q^{\perp} & \\ +(A m)(x, t) \cdot \nabla q+m(x) \nabla p=g & \text { in } Q_{0}, \\ \nabla \cdot(m(x) q)=0 & \text { in } Q_{0}, \\ q=0 & \text { on } \Sigma_{0}, \\ q(\cdot, 0)=w^{0} / m(\cdot) & \text { in } U,\end{cases}
$$

where

$$
g=f+\nabla \cdot(q \nabla m(x))-(A \cdot \nabla m)(x, t) q .
$$

- In order to get energy estimates for the solution of (13), we multiply the differential equation by $q$ and we integrate in $U$. We obtain

$$
\frac{1}{2} \frac{d}{d t} \int_{U} m|q|^{2} d x+\int_{U} m|\nabla q|^{2} d x \leq C\left(\int_{U}|f|^{2} d x d t+\int_{U} m|q|^{2} d x d t\right),
$$

for a positive constant $C$. Here, we have used Young's inequality, combined with the facts that $m \in W^{1, \infty}(U)$ and $0<m_{1}<m(x)<m_{2}$ in $U$. As a consequence, we have that

$$
q \in Y:=L^{2}\left(0, T ; H_{0}^{1}(U)\right) \cap L^{\infty}\left(0, T ; L^{2}(U)\right)
$$

and there exists a positive constant $C>0$ such that

$$
\|q\|_{Y} \leq C\left(\left\|w^{0}\right\|_{L^{2}(U)}+\|f\|_{L^{2}\left(Q_{0}\right)}\right) .
$$


- Now, we multiply the equation in (13) by $q_{t}$. Direct computations give again

$$
\int_{U} m\left|q_{t}\right|^{2} d x+\frac{1}{2} \frac{d}{d t} \int_{U} m|\nabla q|^{2} d x \leq C\left(\int_{U}|f|^{2} d x+\int_{U} m|\nabla q|^{2} d x d t\right),
$$

for some $C>0$. Then, we have shown that

$$
q \in X_{0}:=H^{1}\left(0, T ; L^{2}(U)\right) \cap L^{\infty}\left(0, T ; H_{0}^{1}(U)\right)
$$

and there exists $C>0$ such that

$$
\|q\|_{X_{0}} \leq C\left(\left\|w^{0}\right\|_{H^{1}(U)}+\|f\|_{L^{2}\left(Q_{0}\right)}\right) .
$$

- Finally, we consider the equation of $w$ like a stationary one. In fact, we divide the equation in (13) by $m(x)$ and we consider the pair $(q, p)$ which can be regarded as the solution of a stationary Stokes problem

$$
\begin{cases}-\Delta q+a(x, t) q^{\perp}+A(x, t) \cdot \nabla q+\nabla p=\widetilde{g} & \text { in } Q_{0}, \\ \nabla \cdot q=-(\nabla m) \cdot q & \text { in } Q_{0}, \\ q=0 & \text { on } \Sigma_{0},\end{cases}
$$

with

$$
\widetilde{g}=f / m-q_{t}+2(\nabla m / m) \cdot \nabla q+(\Delta m / m) q-(A \cdot \nabla m / m) q .
$$

Observe that $t$ is watched as a parameter in (16). From (15), we have that $\widetilde{g} \in L^{2}\left(Q_{0}\right)$ and

$$
\|\widetilde{g}\|_{L^{2}\left(Q_{0}\right)} \leq C\left(\left\|w^{0}\right\|_{H^{1}(U)}+\|f\|_{L^{2}\left(Q_{0}\right)}\right) .
$$

Thus, we have a stationary Stokes system for $q$ with right hand side in $L^{2}\left(Q_{0}\right)$ and divergence data in $L^{2}\left(0, T ; H^{1}(U)\right)$ (see (14)), so we know (see, for instance, [15]) that $(q, p) \in L^{2}\left(0, T ; H^{2}(U) \times H^{1}(U)\right)$ and

$$
\|(q, p)\|_{L^{2}\left(0, T ; H^{2}(U) \times H^{1}(U)\right)} \leq C\left(\|\widetilde{g}\|_{L^{2}\left(Q_{0}\right)}+\|q\|_{L^{2}\left(0, T ; H^{1}(U)\right)}\right) .
$$

Using (18), (17), (15) and (14), we finally obtain that $(q, p) \in X \times L^{2}\left(0, T ; H^{1}(U)\right)$ and

$$
\|(q, p)\|_{X \times L^{2}\left(0, T ; H^{1}(U)\right)} \leq C\left(\left\|w^{0}\right\|_{H^{1}(U)}+\|f\|_{L^{2}\left(Q_{0}\right)}\right),
$$

for some $C>0$. From the properties of $m$ and taking into account that $w=$ $m(x, t) q$, we readily deduce (11) and (12) for $(w, p)$.

\subsection{Carleman estimate}

Let us introduce the system

$$
\begin{cases}-\varphi_{t}-\Delta \varphi+a(x, t) \varphi^{\perp}+A(x, t) \cdot \nabla \varphi+m(x) \nabla \pi^{0}=f_{0} & \text { in } Q_{0}, \\ \nabla \cdot \varphi=0 & \text { in } Q_{0}, \\ \varphi=0 & \text { on } \Sigma_{0}, \\ \varphi(\cdot, T)=\varphi^{0} & \text { in } U,\end{cases}
$$


where $\varphi^{0} \in L^{2}(U)$ and $f_{0} \in L^{2}\left(Q_{0}\right)$. Recall that $\varphi^{\perp}$ stands for $\left(-\varphi_{2}, \varphi_{1}\right)$ if $N=2$ and for $\varphi$ if $N>2$.

In order to state the Carleman inequality, we define several weight functions which will be useful in the sequel. Let $\mathcal{O}$ be a nonempty open subset of $U$. The basic weight will be a function $\eta^{0} \in C^{2}(\bar{U})$ satisfying

$$
\eta^{0}>0 \text { in } U, \quad \eta^{0}=0 \text { on } \partial U, \quad\left|\nabla \eta^{0}\right|>0 \text { in } \overline{U \backslash \mathcal{O}_{1}},
$$

where $\mathcal{O}_{1} \subset \subset \mathcal{O}$ is a nonempty open set. The existence of such a function $\eta^{0}$ is proved in [6] and requires $\partial U \in C^{2}$. Then, we introduce

$$
\begin{aligned}
& \alpha(x, t)=\frac{e^{5 / 4 \lambda k\left\|\eta^{0}\right\|_{\infty}}-e^{\lambda\left(k\left\|\eta^{0}\right\|_{\infty}+\eta^{0}(x)\right)}}{t^{4}(T-t)^{4}}, \\
& \xi(x, t)=\frac{e^{\lambda\left(k\left\|\eta^{0}\right\|_{\infty}+\eta^{0}(x)\right)}}{t^{4}(T-t)^{4}}, \\
& \widehat{\alpha}(t)=\min _{x \in \bar{U}} \alpha(x, t)=\frac{e^{5 / 4 \lambda k\left\|\eta^{0}\right\|_{\infty}}-e^{\lambda(k+1)\left\|\eta^{0}\right\|_{\infty}}}{t^{4}(T-t)^{4}}, \\
& \alpha^{*}(t)=\max _{x \in \bar{U}} \alpha(x, t)=\frac{e^{5 / 4 \lambda k\left\|\eta^{0}\right\|_{\infty}}-e^{\lambda k\left\|\eta^{0}\right\|_{\infty}}}{t^{4}(T-t)^{4}}, \\
& \widehat{\xi}(t)=\max _{x \in \bar{U}} \xi(x, t)=\frac{e^{\lambda(k+1)\left\|\eta^{0}\right\|_{\infty}}}{t^{4}(T-t)^{4}}, \quad \xi^{*}(t)=\min _{x \in \bar{U}} \xi(x, t)=\frac{e^{\lambda k\left\|\eta^{0}\right\|_{\infty}}}{t^{4}(T-t)^{4}},
\end{aligned}
$$

where $k>4$ is a fixed real number and $s, \lambda>0$ are real numbers.

Along the proof, we will also use the notation

$$
\begin{aligned}
& I(\varphi)=s^{-1} \iint_{Q_{0}} e^{-2 s \alpha} \xi^{-1}\left(\left|\varphi_{t}\right|^{2}+|\Delta \varphi|^{2}\right) d x d t \\
& +s \lambda^{2} \iint_{Q_{0}} e^{-2 s \alpha} \xi|\nabla \varphi|^{2} d x d t+s^{3} \lambda^{4} \iint_{Q_{0}} e^{-2 s \alpha} \xi^{3}|\varphi|^{2} d x d t .
\end{aligned}
$$

We present the Carleman inequality in the following proposition:

Proposition 1 Let us suppose that $a, A \in W^{1, \infty}\left(0, T ; L^{\infty}(U)\right)$ and $m \in W^{1, \infty}(U)$. Then, there exists a positive constant $C$ depending on $U, \mathcal{O}$ and $T$, such that the solution of (19) satisfies

$$
\begin{aligned}
I(\varphi)+\iint_{Q_{0}} e^{-2 s \alpha}\left|\nabla \pi_{0}\right|^{2} d x d t+s^{2} \lambda^{2} \iint_{Q_{0}} e^{-2 s \alpha} \xi^{2}\left|\pi_{0}\right|^{2} d x d t \\
\leq C s^{7} \lambda^{4}\left(\iint_{Q_{0}} e^{-4 s \widehat{\alpha}+2 s \alpha^{*}}(\widehat{\xi})^{15 / 2}\left|f_{0}\right|^{2} d x d t\right. \\
\left.+\iint_{\mathcal{O} \times(0, T)} e^{-4 \widehat{\alpha}+2 s \alpha^{*}}(\widehat{\xi})^{15 / 2}|\varphi|^{2} d x d t\right),
\end{aligned}
$$

for any $s, \lambda \geq C$. 
The proof estimate (22) will be given in the Appendix, at the end of the paper.

Now, we state some technical results which will be necessary in the sequel of this paper. The first one deals with Carleman inequalities for the heat system:

Lemma 2 Assume that $\partial U \in C^{2}, u^{0} \in L^{2}(U)$ and $g \in L^{2}\left(Q_{0}\right)$. Let $u$ be the solution to the following backwards heat system:

$$
\begin{cases}-u_{t}-\Delta u=g & \text { in } Q_{0}, \\ u=0 & \text { on } \Sigma_{0}, \\ u(\cdot, T)=u^{0} & \text { in } U .\end{cases}
$$

Then there exists a positive constant $C$, depending on $U$ and $\mathcal{O}$, such that

$$
I(u) \leq C\left(s^{3} \lambda^{4} \iint_{\mathcal{O}_{1} \times(0, T)} e^{-2 s \alpha} \xi^{3}|u|^{2} d x d t+\iint_{Q_{0}} e^{-2 s \alpha}|g|^{2} d x d t\right),
$$

for any $s \geq C$ and any $\lambda \geq C$.

The proof of this Carleman inequality can be found in [6]. The fact that $s$ and $\lambda$ can be taken independently (which is essential for the arguments in this paper), can be found in [2].

Remark 3 Observe that the previous lemma also holds when considering exponentials weights of the form $\exp \left\{-r_{0} s \alpha\right\} \quad\left(r_{0}>0\right)$ instead of $\exp \{-2 s \alpha\}$. Of course, this weight function would appear in both sides of the Carleman inequality.

In order to state the next result, we need to introduce a new weight function, namely

$$
\theta(x)=e^{\lambda \eta^{0}(x)} .
$$

We have:

Lemma 3 Let $g \in L^{2}(U)$ be given and let $\phi \in H^{1}(U)$ fulfill the elliptic equation

$$
-\Delta \phi=\nabla \cdot g \text { in } U .
$$

Then there exists $C>0$, depending on $U$ and $\mathcal{O}$, such that

$$
\begin{aligned}
& \int_{U} e^{2 \tau \theta}|\nabla \phi|^{2} d x+\tau^{2} \lambda^{2} \int_{U} e^{2 \tau \theta} \theta^{2}|\phi|^{2} d x \\
& \quad \leq C\left(\tau^{2} \lambda^{2} \int_{\mathcal{O}_{2}} e^{2 \tau \theta} \theta^{2}|\phi|^{2} d x+\tau \int_{U} e^{2 \tau \theta} \theta|g|^{2} d x+\tau^{1 / 2} e^{2 \tau}\|\phi\|_{H^{1 / 2}(\partial U)}^{2}\right)
\end{aligned}
$$

for any $\tau, \lambda \geq C$. Here, $\mathcal{O}_{1} \subset \subset \mathcal{O}_{2} \subset \subset \mathcal{O}$.

The proof of this lemma can be found in [10]. In fact, the inequality stated in that work also has a local integral of $|\nabla \phi|^{2}$ (in $\mathcal{O}_{1}$ ) in the right hand side, but a simple localization argument readily leads to the previous inequality. 


\section{Approximate Controllability}

In this section we prove Theorem 1. As explained in the introduction, we have to prove that for the solutions $\left(\psi, \gamma, \beta_{S}\right)$ of the adjoint system

$$
\begin{cases}-\psi_{t}-\Delta \psi-\partial_{z z}^{2} \psi-\alpha\left(x_{2}\right) \psi^{\perp}+\nabla \beta_{S}=0 & \text { in } Q, \\ \nabla \cdot \psi+\partial_{z} \gamma=0 & \text { in } Q, \\ \psi=0 & \text { on } \Sigma_{l}, \\ \partial_{z} \psi=0, \quad \gamma=0 & \text { on } \Sigma_{S}, \\ B^{*}(\psi)=0, \quad(\psi, \gamma) \cdot n_{b}=0 & \text { on } \Sigma_{b}, \\ \psi(\cdot, T)=\psi^{0} & \text { in } \Omega\end{cases}
$$

with $\psi^{0} \in L^{2}(\Omega)$, the following property is satisfied:

$$
\psi=0 \text { in } \omega \times(0, T) \Rightarrow(\psi, \gamma)=0 \text { in } \Omega \times(0, T) .
$$

First, we decompose $\psi=\bar{\psi}+\widetilde{\psi}$, where $\bar{\psi}$ is the mean value of $\psi$ in the $z$-variable, that is to say:

$$
\bar{\psi}(x, t)=\frac{1}{D(x)} \int_{-D(x)}^{0} \psi(x, z, t) d z .
$$

Then, one can check that the variable $\bar{\psi}$ satisfies the problem

$$
\begin{cases}-\bar{\psi}_{t}-\Delta \bar{\psi}-\alpha\left(x_{2}\right) \bar{\psi}^{\perp}+\nabla \beta_{S}=\ell_{0} & \text { in } S \times(0, T), \\ \nabla \cdot \bar{\psi}=(\nabla D / D) \cdot \bar{\psi} & \text { in } S \times(0, T), \\ \bar{\psi}=0 & \text { on } \partial S \times(0, T), \\ \bar{\psi}(\cdot, T)=\bar{\psi}^{0} & \text { in } S,\end{cases}
$$

where

$$
\bar{\psi}^{0}:=\frac{1}{D(x)} \int_{-D(x)}^{0} \psi^{0}(x, z) d z
$$

and

$$
\ell_{0}=-\frac{1}{D(x)} \partial_{z} \widetilde{\psi}(x,-D(x))-(\Delta D / D) \bar{\psi}(x,-D(x))-2 \nabla D \cdot \nabla \bar{\psi}(x,-D(x))
$$

Notice that the last hypothesis in (3) has been used in order to obtain $\bar{\psi}=0$ on $\partial S \times(0, T)$. by

Let us denote $\zeta=\partial_{z} \psi$. Since $\partial_{z} \psi=\partial_{z} \widetilde{\psi}$ and $\int_{-D(x)}^{0} \widetilde{\psi}=0$, then, $\widetilde{\psi}$ is given

$$
\widetilde{\psi}(x, z, t)=-\int_{z}^{0} \zeta(x, s, t) d s+\frac{1}{D(x)} \int_{-D(x)}^{0} \int_{z}^{0} \zeta(x, s, t) d s d z .
$$


Now, differentiating (25) with respect to $z$, we have that $\zeta$ satisfies the heat equation

$$
-\zeta_{t}-\Delta \zeta-\partial_{z z}^{2} \zeta-\alpha\left(x_{2}\right) \zeta^{\perp}=0 \text { in } Q .
$$

Furthermore, our hypothesis implies that $\zeta=0$ in $\omega \times(0, T)$. From the unique continuation property for the heat equation (which was first proved in [14]), we deduce that $\zeta=0$ in $Q$ and so (28) implies that $\widetilde{\psi}=0$ in $Q$.

Then, the system fulfilled by $\bar{\psi}$ is

$$
\begin{cases}-\bar{\psi}_{t}-\Delta \bar{\psi}-\alpha\left(x_{2}\right) \bar{\psi}^{\perp}+\nabla \beta_{S}=-(\Delta D / D) \bar{\psi}-2 \nabla D \cdot \nabla \bar{\psi} & \text { in } S \times(0, T), \\ \nabla \cdot \bar{\psi}=-(\nabla D / D) \cdot \bar{\psi} & \text { in } S \times(0, T), \\ \bar{\psi}=0 & \text { on } \partial S \times(0, T), \\ \bar{\psi}(\cdot, T)=\bar{\psi}^{0} & \text { in } S .\end{cases}
$$

Finally, in order to prove that $\bar{\psi}=0$ in $S \times(0, T)$ we will use the Carleman inequality proved in Proposition 1 . For this, we define $\widehat{\psi}=D(x) \bar{\psi}$, which satisfies

$$
\begin{cases}-\widehat{\psi}_{t}-\Delta \widehat{\psi}-\alpha\left(x_{2}\right) \widehat{\psi}^{\perp}+D(x) \nabla \beta_{S}=\ell_{1} & \text { in } S \times(0, T), \\ \nabla \cdot \widehat{\psi}=0 & \text { in } S \times(0, T), \\ \widehat{\psi}=0 & \text { on } \partial S \times(0, T), \\ \bar{\psi}(\cdot, T)=\widehat{\psi}^{0} & \text { in } S,\end{cases}
$$

with $\widehat{\psi}^{0}:=D(x) \bar{\psi}^{0}$ and

$$
\ell_{1}=\left(-\Delta D+2 \frac{|\nabla D|^{2}}{D}\right) \widehat{\psi}-2 \nabla D \cdot \nabla \widehat{\psi} .
$$

Now, we are ready to apply Proposition 1 to $\widehat{\psi}$ with $N=2, U=S, \mathcal{O}=P_{S}(\omega)$ (the projection of $\omega$ on $S$ ), $f_{0}=0, m(x)=D(x)$ and

$$
a(x)=\Delta D+2 \frac{|\nabla D|^{2}}{D}, \quad B(x)=2 \nabla D .
$$

As a consequence, we have that there exists a positive constant $C$ depending on $S, \omega$ and $T$, such that such that the solution of $\widehat{\psi}$ satisfies in particular that

$$
I(\widehat{\psi}) \leq C s^{7} \lambda^{4} \iint_{P_{S}(\omega) \times(0, T)} e^{-4 s \widehat{\alpha}+2 s \alpha^{*}}(\widehat{\xi})^{15 / 2}|\widehat{\psi}|^{2} d x d t,
$$

for any $s, \lambda \geq C$. Since $\partial_{z} \psi=\zeta \equiv 0$ and, by hypothesis, $\psi=0$ in $\omega \times(0, T)$, we get that

$$
\psi=0 \text { in } \widetilde{\omega} \times(0, T):=P_{S}(\omega) \times(-D(x), 0) \times(0, T)
$$

and so $\widehat{\psi}=0$ in $P_{S}(\omega) \times(0, T)$. Then, from (31) we readily deduce that $\widehat{\psi}=0$ (hence $\bar{\psi}=0)$ in $S \times(0, T)$. This ends the proof of Theorem 1 . 


\section{Exact Controllability}

In this section we prove Theorem 2 .

With the same notations as in the previous section, we introduce the adjoint system

$$
\begin{cases}-\psi_{t}-\Delta \psi-\partial_{z z}^{2} \psi-\alpha\left(x_{2}\right) \psi^{\perp}+\nabla \beta_{S}=0 & \text { in } Q, \\ \nabla \cdot \psi+\partial_{z} \gamma=0 & \text { in } Q, \\ \psi=0 & \text { on } \Sigma_{l}, \\ \partial_{z} \psi=0, \quad \gamma=0 & \text { on } \Sigma_{S}, \\ \partial_{z} \psi=0, \quad(\psi, \gamma) \cdot n_{b}=0 & \text { on } \Sigma_{b}, \\ \psi(\cdot, T)=\psi^{0} & \text { in } \Omega .\end{cases}
$$

As we observed in the previous section, we have $\psi=\bar{\psi}+\widetilde{\psi}$, where $\bar{\psi}=\widehat{\psi} / D$ and $\widehat{\psi}$ satisfies

$$
\begin{cases}-\widehat{\psi}_{t}-\Delta \widehat{\psi}-\alpha\left(x_{2}\right) \widehat{\psi}^{\perp}+D(x) \nabla \beta_{S}=\ell_{2} & \text { in } S \times(0, T), \\ \nabla \cdot \widehat{\psi}=0 & \text { in } S \times(0, T), \\ \widehat{\psi}=0 & \text { on } \partial S \times(0, T), \\ \bar{\psi}(\cdot, T)=\widehat{\psi}^{0} & \text { in } S,\end{cases}
$$

with

$$
\begin{aligned}
\ell_{2} & =\left(-\Delta D+2 \frac{|\nabla D|^{2}}{D}\right) \widehat{\psi}-2 \nabla D \cdot \nabla \widehat{\psi} \\
& -D(\Delta D) \widetilde{\psi}(x,-D(x))-2 D(\nabla D \cdot \nabla \widetilde{\psi}(x,-D(x))) .
\end{aligned}
$$

Recall that $\widetilde{\psi}$ just depends on $\zeta=\partial_{z} \psi$ and was defined in (28).

First, we apply the Carleman estimate presented in Proposition 1 to $\widehat{\psi}$ as solution of (33) for $U=S, \mathcal{O}=P_{S}(\omega)$, the coefficients $m(x)=D$,

$$
a(x)=\Delta D+2 \frac{|\nabla D|^{2}}{D}, \quad A(x)=2 \nabla D
$$

and

$$
f_{0}=-D(\Delta D) \widetilde{\psi}(x,-D(x))-2 D(\nabla D \cdot \nabla \widetilde{\psi}(x,-D(x))) .
$$

This provides

$$
\begin{aligned}
& I(\widehat{\psi})+\iint_{Q_{0}} e^{-2 s \alpha}\left|\nabla \beta_{S}\right|^{2} d x d t+s^{2} \lambda^{2} \iint_{Q_{0}} e^{-2 s \alpha} \xi^{2}\left|\beta_{S}\right|^{2} d x d t \\
& \leq C s^{7} \lambda^{4}\left(\iint_{Q_{0}} e^{-4 s \widehat{\alpha}+2 s \alpha^{*}}(\widehat{\xi})^{15 / 2}\left(|\widetilde{\psi}|^{2}+|\nabla \widetilde{\psi}|^{2}\right)(x,-D(x)) d x d t\right. \\
& \left.\quad+\iint_{P_{S}(\omega) \times(0, T)} e^{-4 s \widehat{\alpha}+2 s \alpha^{*}}(\widehat{\xi})^{15 / 2}|\widehat{\psi}|^{2} d x d t\right)
\end{aligned}
$$


for any $s, \lambda \geq C$. Looking at the definition of $\widetilde{\psi}$ given in (28), we have that

$$
|\widetilde{\psi}(x,-D(x))|^{2}+|\nabla \widetilde{\psi}(x,-D(x))|^{2} \leq C \int_{-D(x)}^{0}\left(|\zeta|^{2}+|\nabla \zeta|^{2}\right)(x, z, t) d z .
$$

Taking into account that the weight functions $\alpha, \xi, \ldots$ are independent of $z$, we see that

$$
\begin{aligned}
& \iint_{Q_{0}} e^{-4 \widehat{\alpha}+2 s \alpha^{*}}(\widehat{\xi})^{15 / 2}\left(|\widetilde{\psi}|^{2}+|\nabla \widetilde{\psi}|^{2}\right) d x d t \\
& \leq C \iint_{Q} e^{-4 s \widehat{\alpha}+2 s \alpha^{*}}(\widehat{\xi})^{15 / 2}\left(|\zeta|^{2}+|\nabla \zeta|^{2}\right) d x d z d t .
\end{aligned}
$$

With this, we deduce in particular the following from (34):

$$
\begin{gathered}
s^{3} \lambda^{4} \iint_{S \times(0, T)} e^{-2 s \alpha} \xi^{3}|\widehat{\psi}|^{2} d x d t+s \lambda^{2} \iint_{S \times(0, T)} e^{-2 s \alpha} \xi|\nabla \widehat{\psi}|^{2} d x d t \\
\leq C s^{7} \lambda^{4}\left(\iint_{Q} e^{-4 s \widehat{\alpha}+2 s \alpha^{*}}(\widehat{\xi})^{15 / 2}\left(|\zeta|^{2}+|\nabla \zeta|^{2}\right) d x d t\right. \\
\left.\quad+\iint_{P_{S}(\omega) \times(0, T)} e^{-4 \widehat{\alpha}+2 s \alpha^{*}}(\widehat{\xi})^{15 / 2}|\widehat{\psi}|^{2} d x d t\right),
\end{gathered}
$$

for any $s, \lambda \geq C$.

Using the definition of $\widehat{\psi}$ and the relation

$$
\psi(x, \widetilde{z})=\int_{z}^{\widetilde{z}} \partial_{z} \psi(x, s) d x+\psi(x, z), \quad(x, \widetilde{z}) \in S \times \widetilde{\omega}
$$

where $(x, z)$ is the projection point of $(x, \widetilde{z})$ on $\omega$, we have

$$
\int_{P_{S}(\omega)}|\widehat{\psi}|^{2} d x \leq C \int_{\widetilde{\omega}}|\psi|^{2} d x d z \leq C\left(\int_{\widetilde{\omega}}|\zeta|^{2} d x d z+\int_{\omega}|\psi|^{2} d x\right) .
$$

Then, multiplying the last inequality by $e^{-4 s \widehat{\alpha}+2 s \alpha^{*}}(\widehat{\xi})^{15 / 2}$ and integrating in $(0, T)$, we deduce from $(36)$

$$
\begin{aligned}
& s^{3} \lambda^{4} \iint_{S \times(0, T)} e^{-2 s \alpha} \xi^{3}|\widehat{\psi}|^{2} d x d t+s \lambda^{2} \iint_{S \times(0, T)} e^{-2 s \alpha} \xi|\nabla \widehat{\psi}|^{2} d x d t \\
& \quad \leq C s^{7} \lambda^{4} \iint_{Q_{0}} e^{-4 s \widehat{\alpha}+2 s \alpha^{*}}(\widehat{\xi})^{15 / 2}\left(|\zeta|^{2}+|\nabla \zeta|^{2}+|\psi|^{2}\right) d x d t .
\end{aligned}
$$

In order to estimate the terms on $\zeta$, we pretend to use Lemma 2 but this cannot be readily done because of the lack of regularity of our domain $\Omega \in W^{1, \infty}$. Then, let us denote

$$
\bar{D}=\max \{D(x): x \in \bar{S}\} .
$$


We extend our domain $\Omega$ over the surface and below the bottom and we call $\widetilde{\Omega}$ this new domain. In particular, we take $\widetilde{\Omega}$ containing the cylinder

$$
\widetilde{\Omega}_{0}:=\{(x, z): x \in S, z \in(-2 \bar{D}, 1)\}
$$

and satisfying $\widetilde{\Omega} \in W^{3, \infty}$ (this suffices for our purposes). Moreover, we extend the solution $\zeta$ of the heat system satisfied by $\zeta$ by zero over the surface and below the bottom of $\Omega$. We denote $\widetilde{\zeta}$ this extension, which obviously satisfies system

$$
\begin{cases}-\widetilde{\zeta}_{t}-\Delta \widetilde{\zeta}-\partial_{z z}^{2} \widetilde{\zeta}-\alpha\left(x_{2}\right) \widetilde{\zeta}^{\perp}=0 & \text { in } \widetilde{Q}:=\widetilde{\Omega} \times(0, T), \\ \widetilde{\zeta}=0 & \text { on } \widetilde{\Sigma}:=\partial \widetilde{\Omega} \times(0, T), \\ \widetilde{\zeta}(\cdot, T)=\widetilde{\partial_{z} \psi^{0}} & \text { in } \widetilde{\Omega} .\end{cases}
$$

We call the bi-dimensional weight function $\eta$ introduced in (20) associated to the open sets $S$ and $P_{S}(\omega)$ (which defines $\alpha, \xi, \cdots$ ). Now, we consider $\eta_{2}$ (which defines $\left.\alpha_{2}, \xi_{2}, \cdots\right)$ a function satisfying (20) for the open sets $\widetilde{\Omega}$ and $\omega_{0} \subset \subset \omega$ such that

$$
s^{6} \lambda^{2} e^{-4 s \widehat{\alpha}+2 s \alpha^{*}}(\widehat{\xi})^{15 / 2} \leq \xi_{2} e^{-2 s \alpha_{2}} .
$$

In order to do this, it suffices to take a function $\eta_{2}$ such that $\left\|\eta_{2}\right\|_{\infty}<<\|\eta\|_{\infty}$ (that is, $\left\|\eta_{2}\right\|_{\infty}$ is much smaller than $\|\eta\|_{\infty}$ ).

We apply Lemma 2 to $\widetilde{\zeta}$ and for this weight function and we obtain

$$
\begin{aligned}
& s \lambda^{2} \iint_{\widetilde{Q}} e^{-2 s \alpha_{2}} \xi_{2}\left(s^{2} \lambda^{2} \xi_{2}^{2}|\widetilde{\zeta}|^{2}+|\nabla \widetilde{\zeta}|^{2}+\left|\partial_{z} \widetilde{\zeta}\right|^{2}\right) d x d z d t \\
& \leq C s^{3} \lambda^{4} \iint_{\omega_{0} \times(0, T)} e^{-2 s \alpha_{2}} \xi_{2}^{3}|\zeta|^{2} d x d z d t,
\end{aligned}
$$

for any $s, \lambda \geq C$. Let us now define a positive cut-off function $\rho_{0} \in C_{c}^{2}(\omega)$ such that $\rho_{0} \equiv 1$ in $\omega_{0}$. Then,

$$
\begin{aligned}
& \iint_{\omega_{0} \times(0, T)} e^{-2 s \alpha_{2}} \xi_{2}^{3}|\zeta|^{2} d x d z d t \leq \iint_{\omega \times(0, T)} \rho_{0} e^{-2 s \alpha_{2}} \xi_{2}^{3}|\zeta|^{2} d x d z d t \\
& =\iint_{\omega \times(0, T)}\left(\frac{1}{2} \partial_{z z}^{2}\left(e^{-2 s \alpha_{2}} \xi_{2}^{3} \rho_{0}\right)|\psi|^{2}-e^{-2 s \alpha_{2}} \xi_{2}^{3} \rho_{0} \psi \partial_{z z}^{2} \psi\right) d x d z d t \\
& \leq \varepsilon s^{-2} \lambda^{-2} \iint_{Q} e^{-2 s \alpha_{2}} \xi_{2}\left|\partial_{z} \zeta\right|^{2} d x d z d t+C s^{2} \lambda^{2} \iint_{\omega \times(0, T)} e^{-2 s \alpha_{2}} \xi_{2}^{5}|\psi|^{2} d x d z d t,
\end{aligned}
$$

for $s, \lambda \geq C$. Putting this together with (39) and (37) and taking into account 
that $\zeta=\widetilde{\zeta}$ in $\Omega$, we find that

$$
\begin{aligned}
& s^{3} \lambda^{4} \iint_{S \times(0, T)} e^{-2 s \alpha} \xi^{3}|\widehat{\psi}|^{2} d x d t+s \lambda^{2} \iint_{S \times(0, T)} e^{-2 s \alpha} \xi|\nabla \widehat{\psi}|^{2} d x d t \\
& +s \lambda^{2} \iint_{Q} e^{-2 s \alpha_{2}} \xi_{2}\left(s^{2} \lambda^{2}\left(\xi_{2}\right)^{2}|\zeta|^{2}+|\nabla \zeta|^{2}+\left|\partial_{z} \zeta\right|^{2}\right) d x d z d t \\
& \quad \leq C s^{5} \lambda^{6} \iint_{\omega \times(0, T)} e^{-2 s \alpha_{2}} \xi_{2}^{5}|\psi|^{2} d x d z d t .
\end{aligned}
$$

The last step to obtain the desired inequality (8) relies on the expression $\psi=\widehat{\psi} / D+\widetilde{\psi}$. First, using the dissipation in time of the system fulfilled by $(\widehat{\psi}, \zeta)$ (see Lemma 1 ), we deduce that

$$
\begin{aligned}
& \int_{\Omega}|\psi(x, z, 0)|^{2} d x d z \leq C\left(\int_{S}|\widehat{\psi}(x, 0)|^{2} d x+\int_{\Omega}|\widetilde{\psi}(x, z, 0)|^{2} d x d z\right) \\
& \leq C\left(\int_{S}|\widehat{\psi}(x, 0)|^{2} d x+\int_{\Omega}|\zeta(x, z, 0)|^{2} d x d z\right) \\
& \leq C\left(\iint_{S \times(T / 4,3 T / 4)}|\widehat{\psi}(x, t)|^{2} d x d t+\iint_{\Omega \times(T / 4,3 T / 4)}|\zeta(x, z, t)|^{2} d x d z d t\right) \\
& \leq C\left(s^{3} \lambda^{4} \iint_{S \times(0, T)} e^{-2 s \alpha} \xi^{3}|\widehat{\psi}|^{2} d x d t+s^{3} \lambda^{4} \iint_{Q} e^{-2 s \alpha_{2}}\left(\xi_{2}\right)^{3}|\zeta|^{2} d x d z d t\right)
\end{aligned}
$$

which is bounded by the left hand side of (40).

In the last inequality we have used that the weight functions are strictly positive as long as we are 'far away' from $t=0$ and $t=T$. By a similar argument, we can also prove that the integral

$$
\int_{\Omega}|\nabla \psi(x, z, 0)|^{2} d x d z
$$

is also bounded by the left hand side of (40). Thanks to this, to the boundary condition $\gamma=0$ on $\Sigma_{S}$ and to the relation

$$
\partial_{z} \gamma=-\nabla \cdot \psi,
$$

one can also prove that

$$
\int_{\Omega}|\gamma(x, z, 0)|^{2} d x d z
$$

is bounded by the left hand side of (40).

With this, inequality (8) is readily deduced from (41).

\section{Appendix: Proof of Proposition 1}

In this appendix we prove the Carleman estimate stated in proposition 1. 
Let us set $\pi:=m(x) \pi^{0}$. Then, we can rewrite system (19) as follows:

$$
\begin{cases}-\varphi_{t}-\Delta \varphi+a(x, t) \varphi^{\perp}+A(x, t) \cdot \nabla \varphi+\nabla \pi=f_{1} & \text { in } Q_{0}, \\ \nabla \cdot \varphi=0 & \text { in } Q_{0}, \\ \varphi=0 & \text { on } \Sigma_{0}, \\ \varphi(\cdot, T)=\varphi^{0} & \text { in } U,\end{cases}
$$

where $f_{1}$ is given by

$$
f_{1}=f_{0}-(\nabla m / m)(x) \pi
$$

In the rest of the proof we drop the $(x, t)$ sign which indicates the dependence on those variables of the coefficients $a, A$ and $m$.

- Some previous Carleman inequalities

Let us first apply the Carleman inequality for the heat equation to $\varphi$ (see lemma 2), where $f_{1}-\nabla \pi$ is seen as a right hand side. This gives

$$
\begin{aligned}
I(\varphi) \leq & C\left(\iint_{Q_{0}} e^{-2 s \alpha}\left(\left|f_{0}\right|^{2}+|\pi|^{2}+|\nabla \pi|^{2}\right) d x d t\right. \\
& \left.+s^{3} \lambda^{4} \iint_{\mathcal{O}_{1} \times(0, T)} e^{-2 s \alpha} \xi^{3}|\varphi|^{2} d x d t\right),
\end{aligned}
$$

for any $s, \lambda \geq C$. The next step will be to localize the pressure terms. For this, we take the divergence operator in the equation of (42) and we find:

$$
\Delta \pi=\nabla \cdot\left(-a \varphi^{\perp}-A \cdot \nabla \varphi+f_{1}\right) .
$$

To this elliptic equation, we apply lemma 3 and we obtain

$$
\begin{aligned}
\int_{U} e^{2 \tau \theta} \mid \nabla & \left.\pi\right|^{2} d x+\tau^{2} \lambda^{2} \int_{U} e^{2 \tau \theta} \theta^{2}|\pi|^{2} d x \\
\leq & C\left(\tau^{2} \lambda^{2} \int_{\mathcal{O}_{2}} e^{2 \tau \theta} \theta^{2}|\pi|^{2} d x+\tau^{1 / 2} e^{2 \tau}\|\pi\|_{H^{1 / 2}(\partial U)}^{2}\right. \\
& \left.+\tau \int_{U} e^{2 \tau \theta} \theta\left(\left|f_{0}\right|^{2}+|\varphi|^{2}+|\nabla \varphi|^{2}+|\pi|^{2}\right) d x\right)
\end{aligned}
$$

for any $\tau, \lambda \geq C$. The global term in the righthand side concerning $\pi$ is automatically eliminated (for an eventual greater $C$ ). In order to combine this estimate with (43), we recall that $\theta(x)=\exp \left\{\lambda \eta^{0}(x)\right\}$ and that

$$
\alpha(x, t)=\frac{e^{5 / 4 \lambda k\left\|\eta^{0}\right\|_{\infty}}-e^{\lambda\left(k\left\|\eta^{0}\right\|_{\infty}+\eta^{0}(x)\right)}}{t^{4}(T-t)^{4}}=\frac{e^{5 / 4 \lambda k\left\|\eta^{0}\right\|_{\infty}}}{t^{4}(T-t)^{4}}-\frac{\theta e^{\lambda k\left\|\eta^{0}\right\|_{\infty}}}{t^{4}(T-t)^{4}} .
$$

Thus, let us set $\tau=s t^{-4}(T-t)^{-4} e^{\lambda k\left\|\eta^{0}\right\|_{\infty}}$. In particular, observe that

$$
\exp (2 \tau \theta)=\exp \left(2 s \frac{\theta e^{\lambda k\left\|\eta^{0}\right\|_{\infty}}}{t^{4}(T-t)^{4}}\right) .
$$


Then, multiplying (44) by

$$
\exp \left(-2 s t^{-4}(T-t)^{-4} e^{\left.(5 / 4) \lambda k\left\|\eta^{0}\right\|_{\infty}\right)}\right.
$$

and integrating with respect to $t$ in $(0, T)$, we get

$$
\begin{aligned}
& \iint_{Q_{0}} e^{-2 s \alpha}|\nabla \pi|^{2} d x d t+s^{2} \lambda^{2} \iint_{Q_{0}} e^{-2 s \alpha} \xi^{2}|\pi|^{2} d x d t \\
& \leq C\left(s^{2} \lambda^{2} \iint_{\mathcal{O}_{2} \times(0, T)} e^{-2 s \alpha} \xi^{2}|\pi|^{2} d x d t+s^{1 / 2} \int_{0}^{T} e^{-2 s \alpha^{*}}\left(\xi^{*}\right)^{1 / 2}\|\pi\|_{H^{1 / 2}(\partial U)}^{2} d t\right. \\
& \left.\quad+s \iint_{Q_{0}} e^{-2 s \alpha} \xi\left(\left|f_{0}\right|^{2}+|\varphi|^{2}+|\nabla \varphi|^{2}\right) d x d t\right)
\end{aligned}
$$

Combining this inequality with (43), we have

$$
\begin{aligned}
& I(\varphi)+\iint_{Q_{0}} e^{-2 s \alpha}|\nabla \pi|^{2} d x d t+s^{2} \lambda^{2} \iint_{Q_{0}} e^{-2 s \alpha} \xi^{2}|\pi|^{2} d x d t \\
& \leq C\left(s^{2} \lambda^{2} \iint_{\mathcal{O}_{2} \times(0, T)} e^{-2 s \alpha} \xi^{2}\left(|\pi|^{2}+s \lambda^{2} \xi|\varphi|^{2}\right) d x d t\right. \\
& \left.\quad+s^{1 / 2} \int_{0}^{T} e^{-2 s \alpha^{*}}\left(\xi^{*}\right)^{1 / 2}\|\pi\|_{H^{1 / 2}(\partial U)}^{2} d t+s \iint_{Q_{0}} e^{-2 s \alpha} \xi\left|f_{0}\right|^{2} d x d t\right),
\end{aligned}
$$

for $s, \lambda \geq C$.

- Estimate of the trace of the pressure

We define the weight function $\ell_{3}(t)=s^{1 / 4} e^{-s \alpha^{*}}\left(\xi^{*}\right)^{1 / 4}$ and we introduce the new variables

$$
\left(\varphi^{*}, \pi^{*}\right)=\ell_{3}(\varphi, \pi) .
$$

They satisfy

$$
\begin{cases}-\varphi_{t}^{*}-\Delta \varphi^{*}+a \varphi^{*}+B \cdot \nabla \varphi^{*}+\nabla \pi^{*}=\ell_{3} f_{1}-\ell_{3, t} \varphi & \text { in } Q_{0}, \\ \nabla \cdot \varphi^{*}=0 & \text { in } Q_{0}, \\ \varphi^{*}=0 & \text { on } \Sigma_{0}, \\ \varphi^{*}(\cdot, T)=0 & \text { in } U .\end{cases}
$$

We apply to this system strong global estimates for the Stokes system (see, for instance, [15]). In particular, this yields $\pi^{*} \in L^{2}\left(0, T ; H^{1}(U)\right)$ and

$$
\begin{aligned}
& \left\|\pi^{*}\right\|_{L^{2}\left(0, T ; H^{1}(U)\right)}^{2} \leq C\left(s^{1 / 2} \iint_{Q_{0}} e^{-2 s \alpha^{*}}\left(\xi^{*}\right)^{1 / 2}\left(\left|f_{0}\right|^{2}+|\pi|^{2}\right) d x d t\right. \\
& \left.+s^{5 / 2} \iint_{Q_{0}} e^{-2 s \alpha^{*}}\left(\xi^{*}\right)^{3}|\varphi|^{2} d x d t\right)
\end{aligned}
$$


for any $s \geq C$. Here, we have used that $\ell_{3, t} \leq C s^{5 / 4} e^{-s \alpha^{*}}\left(\xi^{*}\right)^{3 / 2}$ for $s \geq C$. Consequently, from (45) we get

$$
\begin{aligned}
& I(\varphi)+\iint_{Q_{0}} e^{-2 s \alpha}|\nabla \pi|^{2} d x d t+s^{2} \lambda^{2} \iint_{Q_{0}} e^{-2 s \alpha} \xi^{2}|\pi|^{2} d x d t \\
& \quad \leq C\left(s^{2} \lambda^{2} \iint_{\mathcal{O}_{2} \times(0, T)} e^{-2 s \alpha} \xi^{2}\left(|\pi|^{2}+s \lambda^{2} \xi|\varphi|^{2}\right) d x d t\right. \\
& \left.\quad+s \iint_{Q_{0}} e^{-2 s \alpha} \xi\left|f_{0}\right|^{2} d x d t\right),
\end{aligned}
$$

for $s, \lambda \geq C$. Here, we have absorbed the terms in the right hand side of (47) concerning $\varphi$ and $\pi$ with $I(\varphi)$ and the third term in the left hand side of (45), respectively.

The last step of the proof consists of estimating the local integral of the pressure:

$$
s^{2} \lambda^{2} \iint_{\mathcal{O}_{2} \times(0, T)} e^{-2 s \alpha} \xi^{2}|\pi|^{2} d x d t \leq s^{2} \lambda^{2} \iint_{\mathcal{O}_{2} \times(0, T)} e^{-2 s \widehat{\alpha} \widehat{\xi}^{2}}|\pi|^{2} d x d t .
$$

First, we choose the pressure $\pi_{0}=\pi / m$ to have null mean-value in the open set $\mathcal{O}_{2}$ and so it suffices to estimate

$$
s^{2} \lambda^{2} \iint_{\mathcal{O}_{2} \times(0, T)} e^{-2 s \widehat{\alpha}} \widehat{\xi}^{2}\left|\nabla \pi_{0}\right|^{2} d x d t
$$

Looking at the equations in (19) and (42), we deduce that

$$
\begin{aligned}
& s^{2} \lambda^{2} \iint_{\mathcal{O}_{2} \times(0, T)} e^{-2 s \hat{\alpha}} \widehat{\xi}^{2}|\pi|^{2} d x d t \\
& \leq C s^{2} \lambda^{2}\left(\iint_{\mathcal{O}_{3} \times(0, T)} e^{-2 s \widehat{\alpha}} \widehat{\xi}^{2}\left(\left|\varphi_{t}\right|^{2}+|\Delta \varphi|^{2}+|\varphi|^{2}+\left|f_{0}\right|^{2}\right) d x d t\right),
\end{aligned}
$$

where $\mathcal{O}_{2} \subset \subset \mathcal{O}_{3} \subset \subset \mathcal{O}$. Here, we have estimated the local integral of $|B \nabla \varphi|^{2}$ in $\mathcal{O}_{2} \times(0, T)$ by those of $|\Delta \varphi|^{2}$ and $|\varphi|^{2}$ in $\mathcal{O}_{3} \times(0, T)$.

Let us work with the terms concerning $\Delta \varphi$ and $\varphi_{t}$.

- Estimate of the terms in $|\Delta \varphi|^{2}$ and $\left|\varphi_{t}\right|^{2}$

Let us set $\left(\widehat{\varphi}, \widehat{\pi}^{0}\right)=\theta_{0}(t)\left(\varphi, \pi^{0}\right)$ with $\theta_{0}(t)=s \lambda e^{-s \widehat{\alpha} \widehat{\xi}}$. Then, $\left(\widehat{\varphi}, \widehat{\pi}^{0}\right)$ fulfills system (19) with $f_{0}$ replaced by $\theta_{0} f_{0}-\theta_{0, t} \varphi$. From (49), we see that the goal is now to estimate the $L^{2}$ norm of $|\Delta \widehat{\varphi}|^{2}$ and $\left|\widehat{\varphi}_{t}\right|^{2}$ in $\mathcal{O}_{3} \times(0, T)$.

Let us introduce now the weight functions

$$
\theta_{1}(t)=s^{5 / 2} \lambda e^{s\left(\alpha^{*}-\widehat{\alpha}\right)}(\widehat{\xi})^{11 / 4}, \quad \text { and } \quad \theta_{2}(t)=\theta_{0}(t) \theta_{1}(t) .
$$

Then, we decompose the previous solution as follows:

$$
\theta_{1}(\widehat{\varphi}, \widehat{\pi})=\left(\widehat{\varphi}^{1}+\widehat{\varphi}^{2}, \widehat{\pi}^{1}+\widehat{\pi}^{2}\right),
$$


where $\left(\widehat{\varphi}^{1}, \widehat{\pi}^{1}\right)$ solves

$$
\begin{cases}-\widehat{\varphi}_{t}^{1}-\Delta \widehat{\varphi}^{1}+a\left(\widehat{\varphi}^{1}\right)^{\perp}+A \cdot \nabla \widehat{\varphi}^{1}+m \nabla \widehat{\pi}^{1}=\theta_{2} f_{0} & \text { in } Q_{0}, \\ \nabla \cdot \widehat{\varphi}^{1}=0 & \text { in } Q_{0}, \\ \widehat{\varphi}^{1}=0 & \text { on } \Sigma_{0}, \\ \widehat{\varphi}^{1}(\cdot, T)=0 & \text { in } U .\end{cases}
$$

and $\left(\widehat{\varphi}_{2}, \widehat{\pi}_{2}\right)$ solves

$$
\begin{cases}-\widehat{\varphi}_{t}^{2}-\Delta \widehat{\varphi}^{2}+a\left(\widehat{\varphi}^{2}\right)^{\perp}+A \cdot \nabla \widehat{\varphi}^{2}+m \nabla \widehat{\pi}^{2}=-\theta_{2, t} \varphi & \text { in } Q_{0}, \\ \nabla \cdot \widehat{\varphi}^{2}=0 & \text { in } Q_{0}, \\ \widehat{\varphi}^{2}=0 & \text { on } \Sigma_{0}, \\ \widehat{\varphi}^{2}(\cdot, T)=0 & \text { in } U .\end{cases}
$$

Consequently, it suffices to estimate both $\left|\theta_{3}^{1 / 2} \Delta \widehat{\varphi}^{i}\right|^{2}$ and $\left|\theta_{3}^{1 / 2} \widehat{\varphi}_{t}^{i}\right|^{2}$ in $\mathcal{O}_{3} \times(0, T)$, for $i=1,2$ with

$$
\theta_{3}(t)=\left(\theta_{1}\right)^{-2}(t)=s^{-5} \lambda^{-2} e^{-2 s\left(\alpha^{*}-\widehat{\alpha}\right)}(\widehat{\xi})^{-11 / 2} .
$$

For $\widehat{\varphi}^{1}$, we use Lemma 1 and we deduce:

$$
\begin{aligned}
& \iint_{\mathcal{O}_{3} \times(0, T)} \theta_{3}\left(\left|\widehat{\varphi}_{t}^{1}\right|^{2}+\left|\Delta \hat{\varphi}^{1}\right|^{2}\right) d x d t \leq \iint_{\mathcal{O}_{3} \times(0, T)}\left(\left|\widehat{\varphi}_{t}^{1}\right|^{2}+\left|\Delta \hat{\varphi}^{1}\right|^{2}\right) d x d t \\
& \leq C \iint_{Q_{0}}\left|\theta_{2}\right|^{2}\left|f_{0}\right|^{2} d x d t .
\end{aligned}
$$

As long as $\widehat{\varphi}^{2}$ is concerned, let $\rho \in C_{c}^{2}(\mathcal{O})$ with $\rho=1$ in $\mathcal{O}_{3}$. Then, integrating by parts, we have

$$
\begin{aligned}
& \iint_{\mathcal{O}_{3} \times(0, T)} \theta_{3}\left|\Delta \widehat{\varphi}^{2}\right|^{2} d x d t \leq \iint_{\mathcal{O} \times(0, T)} \rho \theta_{3}\left|\Delta \widehat{\varphi}^{2}\right|^{2} d x d t \\
& =\iint_{\mathcal{O} \times(0, T)} \theta_{3}\left(\rho \Delta^{2} \widehat{\varphi}^{2}+2 \nabla \rho \cdot \nabla \Delta \widehat{\varphi}^{2}+\Delta \rho \Delta \widehat{\varphi}^{2}\right) \cdot \widehat{\varphi}^{2} d x d t \\
& \leq \varepsilon\left\|\theta_{3} \widehat{\varphi}^{2}\right\|_{L^{2}\left(0, T ; H^{4}(\mathcal{O})\right)}^{2}+C\left\|\widehat{\varphi}^{2}\right\|_{L^{2}(\mathcal{O} \times(0, T))}^{2},
\end{aligned}
$$

where $\varepsilon>0$ is a small constant.

On the other hand, we integrate by parts in the term concerning $\left|\widehat{\varphi}_{t}^{2}\right|^{2}$ and we obtain

$$
\begin{aligned}
& \iint_{\mathcal{O}_{3} \times(0, T)} \theta_{3}\left|\widehat{\varphi}_{t}^{2}\right|^{2} d x d t=\iint_{\mathcal{O}_{3} \times(0, T)}\left(-\theta_{3} \widehat{\varphi}_{t t}^{2} \widehat{\varphi}^{2}+\frac{1}{2}\left(\theta_{3}\right)_{t t}\left|\widehat{\varphi}^{2}\right|^{2}\right) d x d t \\
& \leq \varepsilon\left\|\theta_{3} \widehat{\varphi}^{2}\right\|_{H^{2}\left(0, T ; L^{2}(\mathcal{O})\right)}^{2}+C\left\|\widehat{\varphi}^{2}\right\|_{L^{2}(\mathcal{O} \times(0, T))}^{2} .
\end{aligned}
$$


From (55), (56) and (57) we deduce that

$$
\begin{aligned}
\iint_{\mathcal{O}_{3} \times(0, T)} & \left(\left|\widehat{\varphi}_{t}\right|^{2}+|\Delta \widehat{\varphi}|^{2}\right) d x d t \\
\leq & \varepsilon\left(\left\|\theta_{3} \widehat{\varphi}^{2}\right\|_{H^{2}\left(0, T ; L^{2}(\mathcal{O})\right)}^{2}+\left\|\theta_{3} \widehat{\varphi}^{2}\right\|_{L^{2}\left(0, T ; H^{4}(\mathcal{O})\right)}^{2}\right) \\
& +C\left\|\widehat{\varphi}^{2}\right\|_{L^{2}(\mathcal{O} \times(0, T))}^{2}+C \iint_{Q_{0}}\left|\theta_{2}\right|^{2}\left|f_{0}\right|^{2} d x d t .
\end{aligned}
$$

Next, we will obtain estimates for the terms concerning the higher norms of $\widehat{\varphi}^{2}$. For this, we introduce the new variables $\left(\varphi^{3}, \pi^{3}\right)=\theta_{3}\left(\widehat{\varphi}^{2}, \widehat{\pi}^{2}\right)$, which satisfy

$$
\begin{cases}-\varphi_{t}^{3}-\Delta \varphi^{3}+a\left(\varphi^{3}\right)^{\perp}+A \cdot \nabla \varphi^{3}+m \nabla \pi^{3}=-\theta_{3} \theta_{2, t} \varphi-\theta_{3, t} \widehat{\varphi}^{2} & \text { in } Q_{0}, \\ \nabla \cdot \varphi^{3}=0 & \text { in } Q_{0}, \\ \varphi^{3}=0 & \text { on } \Sigma_{0}, \\ \varphi^{3}(\cdot, T)=0 & \text { in } U .\end{cases}
$$

Due to the exponential decay of $\left(\varphi^{3}, \pi^{3}\right)$ as $t \rightarrow T^{-}$, following the arguments in the proof of Lemma 1 and assuming for a moment that $\theta_{3} \theta_{2, t} \varphi+\theta_{3, t} \widehat{\varphi}^{2} \in X$ (recall that $X$ was defined in (11)), it is not difficult to see that $\left(\varphi^{3}, \pi^{3}\right)$ belongs to the space $X_{1} \times X_{2}$, where

$$
\begin{aligned}
& X_{1}:=L^{2}\left(0, T ; H^{4}(U)\right) \cap H^{2}\left(0, T ; L^{2}(U)\right) \\
& X_{2}:=L^{2}\left(0, T ; H^{3}(U)\right) \cap H^{1}\left(0, T ; H^{1}(U)\right)
\end{aligned}
$$

and

$$
\left\|\left(\varphi^{3}, \pi^{3}\right)\right\|_{X_{1} \times X_{2}} \leq\left\|\left(\theta_{3} \theta_{2, t}+\theta_{3, t} \widehat{\varphi}^{2}\right) \varphi\right\|_{X} .
$$

Observe that from the definitions of $\theta_{2}$ and $\theta_{3}$ given in (50) and (54) respectively, we deduce that

$$
\theta_{3} \theta_{2, t}=s^{-3 / 2} e^{-2 s\left(\alpha^{*}-\widehat{\alpha}\right)}(\widehat{\xi})^{-11 / 2}\left(e^{s \alpha^{*}-2 s \widehat{\alpha}}(\widehat{\xi})^{15 / 4}\right)_{t} .
$$

Let us denote

$$
\theta_{4}(t)=s^{-1 / 4} e^{-s \alpha^{*}}\left(\xi^{*}\right)^{-1 / 2} .
$$

Plugging this into (58), we get

$$
\begin{aligned}
& \iint_{\mathcal{O}_{3} \times(0, T)}\left(\left|\widehat{\varphi}_{t}\right|^{2}+|\Delta \widehat{\varphi}|^{2}\right) d x d t \leq \varepsilon\left(\left\|\theta_{4} \varphi\right\|_{X}^{2}+\left\|\theta_{3, t} \widehat{\varphi}^{2}\right\|_{X}\right) \\
& \quad+C\left\|\widehat{\varphi}^{2}\right\|_{L^{2}(\mathcal{O} \times(0, T))}^{2}+C \iint_{Q_{0}}\left|\theta_{2}\right|^{2}\left|f_{0}\right|^{2} d x d t .
\end{aligned}
$$

$>$ From the definition of $I(\varphi)$, and (51) together with the estimate for $\widehat{\varphi}^{1}(55)$, we have

$$
\begin{aligned}
& \iint_{\mathcal{O}_{3} \times(0, T)}\left(\left|\widehat{\varphi}_{t}\right|^{2}+|\Delta \widehat{\varphi}|^{2}\right) d x d t \\
& \quad \leq \varepsilon I(\varphi)+C\left(\left\|\theta_{2} \varphi\right\|_{L^{2}(\mathcal{O} \times(0, T))}^{2}+\left\|\theta_{2} f_{0}\right\|_{L^{2}\left(Q_{0}\right)}^{2}\right) .
\end{aligned}
$$


Combining this with (48) and (49) and using the definition of $\theta_{2}$ given in (50), we obtain

$$
\begin{gathered}
I(\varphi)+\iint_{Q_{0}} e^{-2 s \alpha}|\nabla \pi|^{2} d x d t+s^{2} \lambda^{2} \iint_{Q_{0}} e^{-2 s \alpha} \xi^{2}|\pi|^{2} d x d t \\
\leq C s^{7} \lambda^{4} \iint_{Q_{0}} e^{-4 s \widehat{\alpha}+2 s \alpha^{*}}(\widehat{\xi})^{15 / 2}\left(\left|f_{0}\right|^{2}+|\varphi|^{2} 1_{\mathcal{O}}\right) d x d t,
\end{gathered}
$$

which readily yields $(22)$.

Acknowledgements: The authors have been partially supported by MECDGI (Spain), under Grant BFM2003-06446.

\section{References}

[1] K. BRYAn, A numerical method for the study of the circulation of the World Ocean, J. Comp. Physics, 4, 347-376 (1969).

[2] E. Fernández-CArA, S. Guerrero, Global Carleman inequalities for parabolic systems and applications to null controllability, to appear in SIAM J. Control Optim.

[3] E. Fernández-Cara, S. Guerrero, O. Yu. Imanuvilov, J.-P. Puel, Local exact controllability of the Navier-Stokes system, J. Math. Pures Appl., Vol 83/12, pp 1501-1542.

[4] P. AzÉrad, F. Guillén. Mathematical justification of the hydrostatic approximation in the primitive equations of geophysical fluid dynamics Siam J. Math. Anal., 33, No. 4 (2001), 847-859.

[5] D. Bresch, F. Guillén-González, N. Masmoudi, M. A. RodríguezBELlido, Asymptotic derivation of a Navier condition for the Primitive Equations, Asymptotic Analysis, 33, no. 3-4, 237-259 (2003).

[6] A. V. Fursikov, O. Yu. Imanuvilov, Controllability of Evolution Equations, Lecture Notes \#34, Seoul National University, Korea, 1996.

[7] A. V. Fursikov, O. Yu. Imanuvilov, Exact local controllability of twodimensional Navier-Stokes equations (Russian) Mat. Sb. 187 (1996), no. 9, 103-138; translation in Sb. Math. 187 (1996), no. 9, 1355-1390.

[8] A. V. Fursikov, O. Yu. Imanuvilov, Exact controllability of the NavierStokes and Boussinesq equations (Russian) Uspekhi Mat. Nauk 54 (1999), no. 3(327), 93-146; translation in Russian Math. Surveys 54 (1999), no. 3, $565-618$.

[9] O. Yu. Imanuvilov, Remarks on exact controllability for the NavierStokes equations, ESAIM Control Optim. Calc. Var. 6 (2001), 39-72. 
[10] O. Yu. Imanuvilov, J.-P. Puel, Global Carleman estimates for weak elliptic non homogeneous Dirichlet problem, Int. Math. Research Notices, 16, 2003, 883-913.

[11] J.-L. Lions, Contrôlabilité Exacte, Stabilisation et Perturbations de Systèmes Distribués, Tomes 1 \& 2. Masson, RMA 8 \& 9. Paris 1988.

[12] J.-L. Lions, R. Temam, S. Wang, On the equations of the Large Scale Ocean, Nonlinearity, 5, 1007-1053 (1992).

[13] J. Pedlosky, Geophysical fluid dynamics, Springer-Verlag, (1982).

[14] J.-C. SAut, B. Scheurer, Unique continuation for some evolution equations, J. Differential Equations 66 (1987), no. 1, 118-139.

[15] R. Temam, Navier-Stokes equations. Theory and numerical analysis. Studies in Mathematics and its applications, 2; North Holland Publishing Co., Amsterdam-New York-Oxford (1977).

[16] M. Ziane, Regularity Results for Stokes Type Systems. Applicable Analysis, Vol. 58, 263-292 (1995). 